\title{
Multi-Stage Pricing Game for Collusion-Resistant Dynamic Spectrum Allocation
}

\author{
Zhu Ji and K. J. Ray Liu, Fellow, IEEE
}

\begin{abstract}
In order to fully utilize scarce spectrum resources, dynamic spectrum allocation becomes a promising approach to increase the spectrum efficiency for wireless networks. However, the collusion among selfish network users may seriously deteriorate the efficiency of dynamic spectrum sharing. The network users' behaviors and dynamics need to be taken into consideration for efficient and robust spectrum allocation. In this paper, we model the spectrum allocation in wireless networks with multiple selfish legacy spectrum holders and unlicensed users as multi-stage dynamic games. In order to combat user collusion, we propose a pricing-based collusion-resistant dynamic spectrum allocation approach to optimize overall spectrum efficiency, while not only keeping the participating incentives of the selfish users but also combating possible user collusion. The simulation results show that the proposed scheme achieves high efficiency of spectrum usage even with the presence of severe user collusion.
\end{abstract}

Index Terms-Game theory, auction theory, cognitive radio, spectrum management, wireless networks.

\section{INTRODUCTION}

$\mathbf{T}$ HE DEMAND for wireless spectrum use has been growing rapidly with the dramatic development of the mobile telecommunication industry in the last decades. In order to fully utilize the scarce spectrum resources, with the development of cognitive radio technologies, dynamic spectrum access becomes a promising approach to increase the efficiency of spectrum usage, which allows unlicensed wireless users to dynamically access the licensed bands from legacy spectrum holders based on leasing agreements. This new wireless networking paradigm, dynamic spectrum access, is also referred to as Next Generation wireless networks [1], [2].

Cognitive radios have the potential to provide the wireless devices with various capabilities, such as frequency agility, adaptive modulation, transmit power control and localization. The advances of cognitive radio technologies make more efficient and intensive spectrum access possible on a negotiated or an opportunistic basis. The FCC has begun to consider more flexible and comprehensive use of available spectrum [3]. The NeXt Generation program of DARPA also aims to dynamically redistribute the allocated spectrum based on cognitive radio technologies [1]. Therefore, great attentions have

Manuscript received March 2, 2007; revised September 1, 2007. This paper has been presented in part at IEEE Symposium on New Frontiers in Dynamic Spectrum Access Networks (DySPAN), Dublin, Ireland, April 2007.

Zhu Ji is with Qualcomm Incorporated, San Diego, CA 92121 USA (e-mail: zji@qualcomm.com).

K. J. Ray Liu is with the Department of Electrical and Computer Engineering and Institute for Systems Research, University of Maryland, College Park, MD 20742 USA (e-mail: kjrliu@umd.edu).

Digital Object Identifier 10.1109/JSAC.2008.080116. been drawn to explore the dynamic spectrum access systems [4], [5]. Traditionally, network-wide spectrum assignment is carried out by a central server, namely, spectrum broker [6], [7]. Recently, distributed spectrum allocation approaches [8], [9] have been studied to enable efficient spectrum sharing only based on local observations.

From economical point of view, the deregulation of spectrum use further encourages market mechanisms for implementing efficient spectrum allocation. Researchers have already started to study dynamic spectrum access via pricing and auction mechanisms [10]-[15]. In [10], the authors proposed an auction-based mechanism to efficiently share spectrum among the users in interference-limited systems. In [11], the price of anarchy was analyzed for spectrum sharing in WiFi networks. A demand responsive pricing framework was proposed in [12] to maximize the profits of legacy spectrum operators while considering the users' response model to the operators' pricing strategy. In [13], the authors considered multi-unit sealed-bid auction for efficient spectrum allocation. In [14], a belief-assisted distributive pricing algorithm was proposed to achieve efficient dynamic spectrum allocation.

Although the existing dynamic spectrum access schemes have achieved some success on enhancing the spectrum efficiency through distributive design and market mechanisms, in order to have robust dynamic spectrum sharing systems, some basic challenges still remain unanswered. First, considering the spectrum dynamics and lack of centralized authority, the spectrum allocation needs to distributively adapt to the dynamics of wireless networks due to node mobility, channel variations or varying wireless traffic based on locally observed information. From the game theoretical point of view, the spectrum allocation needs to be studied in a multi-stage dynamic game framework instead of the static game approach. Second, with the emerging applications of mobile ad hoc networks envisioned in civilian usage, the users may be selfish and aim to maximize their own interests. In dynamic spectrum allocation scenarios, the selfish users' cheating behaviors need to be well handled for having a robust spectrum allocation scheme. Especially, the collusive behavior of selfish users [16], [17], a prevalent threat to efficient dynamic spectrum allocation, has been generally overlooked and needs to be extensively studied. Therefore, novel spectrum allocation approaches need to be developed considering the spectrum dynamics and countermeasures to the users' collusive behaviors.

Considering a general network scenario in which multiple primary users (legacy spectrum holders) and secondary users (unlicensed users) coexist, primary users attempt to sell unused spectrum resources to secondary users for monetary gains 
while secondary users try to acquire spectrum usage permissions from primary users to achieve certain communication goals, which generally introduces reward payoffs for them. In this paper, we model the spectrum allocation as multistage dynamic games and propose an efficient pricing-based distributive collusion-resistant dynamic spectrum allocation approach to optimize the overall spectrum efficiency, meanwhile, keeping the participating incentives of the users based on double-auction rules. The main contributions of this paper are multi-fold. First, by modeling the spectrum sharing as a multi-stage dynamic pricing game, we are able to coordinate the spectrum allocation among primary and secondary users through a bilateral pricing process to maximize the utilities of both primary and secondary users according to the spectrum dynamics. More importantly, our study is further focused on the countermeasures to selfish users' collusive behaviors. A distributed collusion-resistant dynamic pricing approach with optimal reserve prices is designed to achieve efficient spectrum allocation while combating the user collusion. The pricing overhead can be largely decreased by introducing a belief function for each user to help decision-making. Moreover, the Nash Bargain Solution is applied for deriving the performance lower bound of the proposed scheme considering the presence of user collusion.

The remainder of this paper is organized as follows: The system model of dynamic spectrum allocation is described in Section II. In Section III, we formulate the spectrum allocation as pricing games based on the system model. In Section IV, a collusion-resistant dynamic pricing approach is proposed to achieve efficient spectrum allocation while combating user collusion. The simulation studies are provided in Section V. Finally, Section VI concludes this paper.

\section{SySTEM MOdEL}

We consider the wireless networks where multiple primary users and secondary users operate simultaneously, which may represent various network scenarios. For instance, the primary users can be the spectrum broker connected to the core network and the secondary users are the base stations equipped with cognitive radio technologies; or the primary users are the access points of a mesh network and the secondary users are the mobile devices. On one hand, considering that the authorized spectrum of primary users may not be fully utilized over time, they prefer to lease the unused channels to the secondary users for monetary gains. On the other hand, since the unlicensed spectrums become more and more crowded, the secondary users may try to lease some unused channels from primary users for more communication gains by providing leasing payments.

In our system model, we assume all users are selfish and rational, that is, their objectives are to maximize their own utilities, not to cause damage to other users. However, users are allowed to cheat whenever they believe cheating behaviors can help them to increase their utilities. Note that not only the cheating behaviors from a single selfish user is possible, but also the collusive cheating behaviors among several selfish users will pose threats to efficient spectrum allocation. Generally speaking, in order to acquire the spectrum licenses from regulatory bodies such as FCC, the primary users have certain operating costs. With regard to secondary users, in order to have the rewards of achieving certain communication goals, they want to utilize more spectrum resources.

Specifically, we consider the collection of the available spectrums from all primary users as a spectrum pool, which totally consists of $N$ non-overlapping channels. Assume there are $J$ primary users and $K$ secondary users, indicated by the set $\mathbf{P}=\left\{p_{1}, p_{2}, \ldots, p_{J}\right\}$ and $\mathbf{S}=\left\{s_{1}, s_{2}, \ldots, s_{K}\right\}$, respectively. We represent the channels authorized to primary user $p_{i}$ using a vector $\mathbf{A}_{i}=\left\{a_{i}^{j}\right\}_{j \in\left\{1,2, \ldots, n_{i}\right\}}$, where $a_{i}^{j}$ represents the channel index in the spectrum pool and $n_{i}$ is the total number of channels belonging to user $p_{i}$. Define $\mathbf{A}$ as the set of all the channels in the spectrum pool. Moreover, denote the acquisition costs of user $p_{i}$ 's channels as the vector $\mathbf{C}_{i}=\left\{c_{i}^{a_{i}^{j}}\right\}_{j \in\left\{1,2, \ldots, n_{i}\right\}}$, where the $j$ th element represents the acquisition cost of the $j$ th channel in $\mathbf{A}_{i}$. For simplicity, we write $c_{i}^{a_{i}^{j}}$ as $c_{i}^{j}$. Denote $\mathbf{C}$ as the set of the costs of all spectrum channels. As for secondary user $s_{i}$, we define her/his payoff vector as $\mathbf{V}_{i}=\left\{v_{i}^{j}\right\}_{j \in\{1,2, \ldots, N\}}$, where the $j$ th element is the reward payoff if this user successfully leases the $j$ th channel in the spectrum pool.

\section{Pricing Game Model}

\section{A. Game Settings for Dynamic Spectrum Allocation}

Based on the system model in the previous section, we are able to define the utility functions of the players in our dynamic game. Specifically, if primary user $p_{i}$ reaches agreements of leasing all or part of her/his channels to secondary users, the utility function of this primary user can be written as follows.

$$
U_{p_{i}}\left(\phi_{\mathbf{A}_{i}}, \alpha_{i}^{\mathbf{A}_{i}}\right)=\sum_{j=1}^{n_{i}}\left(\phi_{a_{i}^{j}}-c_{i}^{j}\right) \alpha_{i}^{a_{i}^{j}},
$$

where $\phi_{\mathbf{A}_{i}}=\left\{\phi_{a_{i}^{j}}\right\}_{j \in\left\{1,2, \ldots, n_{i}\right\}}$ and $\phi_{a_{i}^{j}}$ is the payment that user $p_{i}$ obtains from the secondary user by leasing the channel $a_{i}^{j}$ in the spectrum pool. Note that $\alpha_{i}^{\mathbf{A}_{i}}=\left\{\alpha_{i}^{a_{i}^{j}}\right\}_{j \in\left\{1,2, \ldots, n_{i}\right\}}$ and $\alpha_{i}^{a_{i}^{j}} \in\{0,1\}$ which indicates if the $j$ th channel of user $p_{i}$ has been allocated to a secondary user or not. For simplicity, we denote $\alpha_{i}^{a_{i}^{j}}$ as $\alpha_{i}^{j}$. Similarly, the utility function of secondary user $s_{i}$ can be modeled as follows.

$$
U_{s_{i}}\left(\phi_{\mathbf{A}}, \beta_{i}^{\mathbf{A}}\right)=\sum_{j=1}^{N}\left(v_{i}^{j}-\phi_{j}\right) \beta_{i}^{j},
$$

where $\phi_{\mathbf{A}}=\left\{\phi_{j}\right\}_{j \in\{1,2, \ldots, N\}}, \beta_{i}^{\mathbf{A}}=\left\{\beta_{i}^{j}\right\}_{j \in\{1,2, \ldots, N\}}$. Note that $\beta_{i}^{j} \in\{0,1\}$ illustrates if secondary user $s_{i}$ successfully leases the $j$ th channel in the spectrum pool or not.

From the above discussion, we can see that the players may have conflicting interests with each other. Specifically, the primary users want to earn as much payments as possible by leasing the unused channels and the secondary users aim to accomplish their communication goals by providing the least possible payments for leasing the channels. Moreover, the spectrum allocation involves multiple channels over time. Therefore, the spectrum users involved in the spectrum allocation process construct a multi-stage non-cooperative pricing game [16], [18]. Moreover, the selfish users will not reveal 
their private information to others unless some mechanisms have been applied to guarantee that it is not harmful to disclose the private information. In general, such non-cooperative game with incomplete information is complex and difficult to study as the players do not know the perfect strategy profile of others. Nevertheless, based on our game setting, the welldeveloped auction theory [17] can be applied to formulate and analyze our pricing game.

In auction games [17], according to an explicit set of rules, the principles (auctioneers) determine resource allocation and prices on the basis of bids from the agents (bidders). In our spectrum allocation pricing game, the primary users (principles) attempt to sell the unused channels to the secondary users and the secondary users (bidders) compete with each other to buy the permission of using primary users' channels. In our pricing game, multiple primary and secondary users coexist, which indicates the double auction scenario [17], [19]. It means that not only the secondary users but also the primary users need to compete with each other to make the beneficial transactions possible by eliciting their willingness of the payments in the forms of bids or asks. Specifically, the most important property of double auction mechanism is its high efficiency such as in the New York Stock Exchange (NYSE) or Chicago Merchandize Exchange (CME). However, in our spectrum allocation games, the collusive cheating behaviors can seriously deteriorate the game outcomes by forming cartels to suppress competition among users and distort the supply and demand relationships. Moreover, due to the dynamic property of spectrum resources, it becomes more difficult for the network users to distinguish if the spectrum pricing variations come from the user collusion or the changing spectrum dynamics. Also, unlike NYSE or CME, either powerful centralized authorities can be pre-assumed or the bandwidth of control channels is very limited. Therefore, we aim to develop an efficient pricing approach for spectrum allocation not only combating user collusion but also adapting to spectrum dynamics distributively.

\section{B. Static Pricing Game and Competitive Equilibrium}

Assume that the available channels from the primary users are leased for usage of certain time period $T$. Also, we assume that the cost of the primary users and reward payoffs of the secondary users remain unchanged over this period. Before this spectrum sharing period, we define a trading period $\tau$, within which the users exchange their information of bids and asks to achieve agreements of spectrum usage. The time period $T+\tau$ is considered as one stage in our pricing game. We first study the interactions of the players in static pricing games. Note that the users' goals are to maximize their own payoff functions. As for the primary users, the optimization problem can be written as follows.

$$
O\left(p_{i}\right)=\max _{\phi_{\mathbf{A}_{i}, \alpha_{i}}^{\mathbf{A}_{i}}} U_{p_{i}}\left(\phi_{\mathbf{A}_{i}}, \alpha_{i}^{\mathbf{A}_{i}}\right), \quad \forall i \in\{1,2, \ldots, J\}
$$

$$
\begin{array}{r}
\text { s.t. } U_{\hat{s}_{a_{i}^{j}}}\left(\left\{\phi_{-a_{i}^{j}}, \phi_{a_{i}^{j}}\right\}, \beta_{i}^{\mathbf{A}}\right) \geq U_{\hat{s}_{a_{i}^{j}}}\left(\left\{\phi_{-a_{i}^{j}}, \tilde{\phi}_{a_{i}^{j}}\right\}, \beta_{i}^{\mathbf{A}}\right), \\
\hat{s}_{a_{i}^{j}} \neq 0, a_{i}^{j} \in \mathbf{A}_{i} .
\end{array}
$$

where $\tilde{\phi}_{a_{i}^{j}}$ is any feasible payment and $\phi_{-a_{i}^{j}}$ is the payment vector excluding the element of the payment for the channel $a_{i}^{j}$. Note that $\hat{s}_{a_{i}^{j}}$ is defined as follows.

$$
\hat{s}_{a_{i}^{j}}= \begin{cases}s_{k} & \text { if } \beta_{k}^{a_{i}^{j}}=1, \\ 0 & \text { if } \beta_{k}^{a_{i}^{j}}=0, \forall k \in\{1,2, \ldots, K\} .\end{cases}
$$

Thus, (4) is the incentive compatible constraint [17]. It means that the secondary users have incentives to provide the optimal payment because they cannot have extra gains by unilaterally cheating on the primary users. Similarly, the optimization problem can be written for the secondary users as follows.

$$
\begin{array}{r}
O\left(s_{i}\right)=\max _{\phi_{\mathbf{A}}, \beta_{i}^{\mathbf{A}}} U_{s_{i}}\left(\phi_{\mathbf{A}}, \beta_{i}^{\mathbf{A}}\right), \quad \forall i \in\{1,2, \ldots, K\} \\
\text { s.t. } U_{\hat{p}_{j}}\left(\left\{\phi_{-j}, \phi_{j}\right\}, \beta_{i}^{\mathbf{A}}\right) \geq U_{\hat{p}_{j}}\left(\left\{\phi_{-j}, \tilde{\phi}_{j}\right\}, \beta_{i}^{\mathbf{A}}\right), \\
\hat{p}_{j} \neq 0, \beta_{i}^{j}=1 .
\end{array}
$$

where $\hat{p}_{j}$ is defined as

$$
\hat{p}_{j}= \begin{cases}p_{k} & \text { if } \beta_{i}^{j}=1, j \in \mathbf{A}_{k}, \alpha_{k}^{j}=1 \\ 0 & \text { otherwise, } \forall k \in\{1,2, \ldots, J\} .\end{cases}
$$

Note that $\hat{p}_{j}$ represents the primary users who have a channel leased to secondary user $s_{i}$. Similarly, (7) is the incentive compatible constraint for the primary users, which guarantees that the primary user will give the usage permission of their channels to the secondary users so that they can receive the optimal payments.

From (3) and (6), we can see that in order to obtain the optimal allocation and payments, a multi-objective optimization problem needs to be solved, which becomes extremely complicated due to our game setting that only involves incomplete information. Thus, in order to make this problem tangible, we analyze it from the game theoretical point of view. Considering the double auction scenarios of our pricing game, Competitive Equilibrium (CE) [19] is a well-known theoretical prediction of the outcomes. It is the price at which the number of buyers willing to buy is equal to the number of sellers willing to sell. Alternatively, CE can also be interpreted as where the supply and demand match [17]. We describe the supply and demand functions of spectrum resources in Figure 1. Note that CE is also proved to be Pareto optimal in stationary double auction scenarios [17].

\section{Multi-Stage Dynamic Pricing Game for Spectrum Alloca- tion}

Considering spectrum dynamics due to mobility, channel variations or wireless traffic variations, the secondary users' reward payoffs and primary users' costs may change over time or spectrum. Thus, $c_{i}^{j}$ and $v_{i}^{j}$ need to be considered as random variables in dynamic scenarios. Without loss of generality, we assume the homogeneous game settings for the statistics of $c_{i}^{j}$ and $v_{i}^{j}$, which satisfy the probability density functions (PDF) $f_{c}(c)$ and $f_{v}(v)$, respectively. Therefore, considering dynamic network conditions, we further model the spectrum sharing as a multi-stage dynamic pricing game. Let $\gamma$ be the discount factor of our multi-stage pricing game. Based on (3) and (6), 


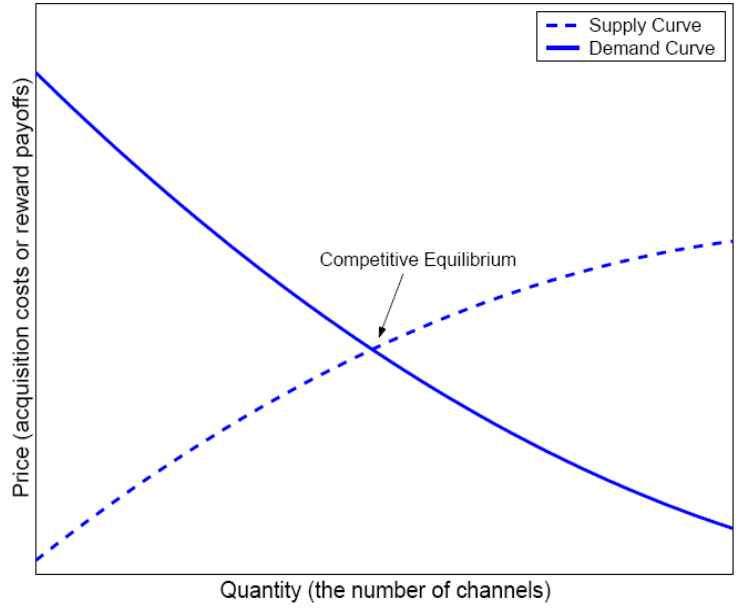

Fig. 1. Illustration of supply and demand functions.

the objective functions for the primary users and secondary users can be rewritten as follows.

$$
\begin{gathered}
\tilde{O}\left(p_{i}\right)=\max _{\phi_{\mathbf{A}_{i}, t}, \alpha_{i, t}^{\mathbf{A}_{i}}} E_{c_{i}^{j}, v_{i}^{j}}\left[\sum_{t=1}^{\infty} \gamma^{t} \cdot U_{p_{i}, t}\left(\phi_{\mathbf{A}_{i}, t}, \alpha_{i, t}^{\mathbf{A}_{i}}\right)\right], \\
\tilde{O}\left(s_{i}\right)=\max _{\phi_{\mathbf{A}, t, \beta_{i, t}^{\mathbf{A}}}} E_{c_{i}^{j}, v_{i}^{j}}\left[\sum_{t=1}^{\infty} \gamma^{t} \cdot U_{s_{i}, t}\left(\phi_{\mathbf{A}, t}, \beta_{i, t}^{\mathbf{A}}\right)\right],
\end{gathered}
$$

where the subscript $t$ indicates the $t$ th stage of the multistage game. Generally speaking, there may exist some overall constraints of spectrum sharing such as each secondary user's total budget for leasing spectrum resources or each primary user's total available spectrum supply. Under these constraints, the above problem can be further solved using dynamic programming process as in [14], [20]. However, the major difficulties of robust and efficient spectrum allocation lie in that how to efficiently combat the selfish users' cheating behaviors especially user collusion only based on local information.

\section{Collusion-Resistant Dynamic Spectrum ALLOCATION}

In this section, we first discuss the impact of user collusion on auction-based dynamic spectrum allocation approaches. Then, we study collusion-resistant dynamic spectrum allocation for two simplified scenarios: (1) multiple secondary users and one primary user (MSOP); (2) one secondary user and multiple primary users (OSMP). Further, we extend our study to a more generalized spectrum allocation scenario with multiple primary users and multiple secondary users (MSMP). Note that the collusion-resistant spectrum allocation in this section is studied under the multi-stage pricing game framework discussed in the previous section.

\section{A. User Collusion in Auction-Based Spectrum Allocation}

In order to have a robust dynamic spectrum allocation mechanism in wireless networks with selfish users, the cheating behaviors of selfish users need to be well studied and

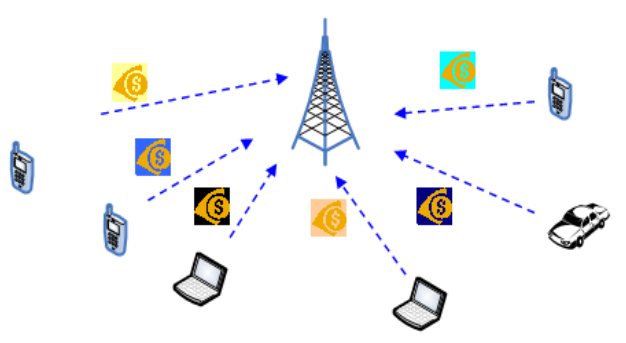

Fig. 2. No collusion in pricing-based dynamic spectrum allocation.

counteracted. Otherwise, the spectrum allocation mechanism may become unsustainable and lead to unpredictable outcomes. On one hand, spectrum allocation can be generally regarded to be similar to generic medium access control (MAC) problems in existing systems and studied from the perspective of wireless resource allocation [7]-[9]. On the other hand, efficient spectrum allocation can be achieved by studying it from the perspective of the driving economic force and mechanisms [12]-[14]. Therefore, the unique property of dynamic spectrum allocation imposes new challenges on its mechanism design against cheating behaviors. Basically, all the cheating behaviors related to MAC problems in wireless system still threaten the functionalities of spectrum sharing mechanisms. More importantly, wireless spectrum becomes a scarce resource and has huge economical potential, which can only be exploited through efficient pricing-based market designs. Thus, the cheating threats on these market designs make the robust dynamic spectrum access a even more complicated problem. Since the cheating behaviors on MAC protocols can still be solved using traditional countermeasures and the auction mechanisms has the incentive-compatible property for each single user, we will focus our study on efficient collusionresistant dynamic spectrum allocation mechanism.

Although incentive-compatibility can be assured in most auction-based dynamic spectrum allocation approaches such as the optimal auction [17], [20] or Vickrey auction [17], which indicates that no selfish user will cheat on the auction mechanism unilaterally, one prevalent cheating behavior, the bidding collusion among users, has been generally overlooked. To be specific, the bidders (or sellers) act collusively and engage in bid rigging with a view to obtaining lower prices (or higher prices). The resulting arrangement is called the bidding ring. In the scenarios of auction-based spectrum allocation, the bidding ring among the primary users (or secondary users) will result in increasing their utilities by collusively leasing the spectrum channels at a higher price (or at a lower price). Considering the spectrum dynamics caused by wireless channel variations, user mobility or varying wireless traffic, it becomes difficult to tell if the price variation comes from possible bidding collusion or the varying demand and supply of spectrum resources. Hence, traditional auctionbased spectrum allocation mechanisms become vulnerable and unstable with the presence of collusive behaviors.

In Figure 2 and Figure 3, we illustrate a snapshot of pricingbased dynamic spectrum access networks where there is no user collusion and exists user collusion, respectively. In the 


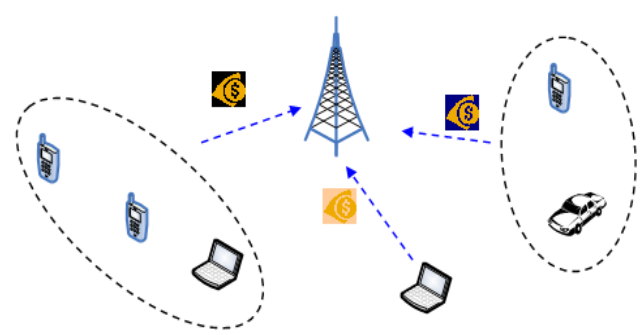

Fig. 3. User collusion in pricing-based dynamic spectrum allocation.

above figures, we consider the primary base station as the primary user and the unlicensed mobile users as the secondary users. When there is no user collusion as in Figure 2, the pricing interactions between the primary user and secondary users lead to efficient spectrum allocation. When there exist several bidding rings as in Figure 3, each bidding ring will elicit only one effective bid for spectrum resources, which distorts the supply and demand of spectrum resources and yields inefficient spectrum allocation. Further, in the extreme case that all secondary users collude with each other, arbitrary low bid price will become eligible. Thus, collusion-resistant dynamic spectrum allocation is important for efficient next generation wireless networking.

In the scenarios of traditional open ascending price, i.e., English auction (or reverse English auction) [17], there is one seller and multiple buyers (or one buyer and multiple sellers). In order to combat the bidding ring, the seller (or buyer) can enhance their utilities by setting proper reserve prices as in [17] based on the size of the bidding ring, i.e, the number of collusive users, and the statistics of each user's true value. However, in our scenarios of dynamic spectrum allocation with multiple primary and secondary users having only local information, either the number of collusive users are not available or the determination of reserve price becomes very complicated given limited imperfect information. Therefore, how to design efficient collusion-resistant dynamic spectrum allocation mechanisms becomes an imminent and crucial task.

\section{B. MSOP and OSMP Scenarios}

In this part, we develop the robust dynamic spectrum allocation mechanisms against user collusion in the scenarios of MSOP and OSMP.

Considering there are one primary user and multiple secondary users in a snap shot of wireless networks, we assume the system model as in Section II except that only one primary user $p_{i}$ is available for providing spectrum leasing services. The standard open ascending price auction is chosen for the secondary users to compete for the spectrum resources, which is theoretically equivalent to sealed-bid second-price auction [17]. Here, the presence of user collusion among secondary users may generate extra utilities for the collusive users by suppressing competition for spectrum resources. Due to the network dynamics and imperfect available information, neither the primary user can make a credible assumption about the presence of user collusion or the number of collusive users, nor there exist trust-worthy anti-cartel authorities in the network. Therefore, the only instrument giving the primary user possible leverage against collusion is to set an optimal reserve price. In the rest of this part, we first derive the theoretical optimal reserve price for our spectrum allocation game similar to [17]. Then, by considering the properties of our spectrum allocation game such as unknown number of collusive users and imperfect/local bidding information, a collusion-resistant dynamic spectrum allocation mechanism is developed to efficiently allocate spectrum resources while combating collusive cheating behaviors.

Specifically, we assume that $K$ secondary users are divided into $K_{r}$ bidding rings and the size of the $k$ th bidding ring is $m_{k}$. Note that $\sum_{k=1}^{K_{r}} m_{k}=K, m_{k} \geq 1$. Basically, the collusion among the secondary users within each bidding ring does not affect the strategies of users out of the bidding ring. Further, the bidding ring can be represented by the collusive secondary user with the highest reward payoff [17]. The other collusive users only submit non-serious bids at or below the reserve price, which substantially limits the competition among secondary users. Thus, instead of $K$ effective competing secondary users, only $K_{r}$ effective users should be considered for bidding spectrum resources. Assume the equivalent reward payoff of the $k$ th bidding ring is $\nu_{m_{k}}^{a_{i}^{j}}$, the highest reward payoff among $m_{k}$ collusive users for the $a_{i}^{j}$ th channel in the spectrum pool. Thus, the payoff vector for effective users can be represented as $\left\{\nu_{1}^{a_{i}^{j}}, \nu_{2}^{a_{i}^{j}}, \ldots, \nu_{K_{r}}^{a_{i}^{j}}\right\}$. Note that we omit the superscript $a_{i}^{j}$ in the following parts for simplicity if the spectrum assignment is only considered for one specific channel. Further, let the highest and second highest reward payoff among all effective secondary users to be $v_{(1)}$ and $v_{(2)}$, respectively.

In order to combat the collusive behaviors of secondary users, the primary user needs to set a reserve price, which means its spectrum resources won't be sold lower than the reserve price. Considering the theoretical equivalence of open ascending price auction and second-price auction, we then study the optimal reserve price for second-price auction setting in our spectrum allocation game. Let the optimal reserve price to be $\phi_{r, p_{i}}$. Then, the spectrum channel can be leased by $p_{i}$ if and only if $v_{(1)}>\phi_{r, p_{i}}$. Moreover, if $v_{(2)}>\phi_{r, p_{i}}$, the spectrum channel is leased for $v_{(2)}$; otherwise, it is leased at the reserve price $\phi_{r, p_{i}}$. Let $F_{v_{(1)}}(x)$ and $F_{v_{(2)}}(x)$ denote the cumulative distribution functions (CDF) of $v_{(1)}$ and $v_{(2)}$, respectively. Let $f_{v_{(1)}}(x)$ and $f_{v_{(2)}}(x)$ denote the probability density functions (PDF) of $v_{(1)}$ and $v_{(2)}$, respectively. Now, the expected utility gain of the primary user with reserve price $\phi_{r, p_{i}}$ by leasing her/his $j$ th channel can be written as

$$
\begin{aligned}
E_{\mathbf{V}_{i}, c_{i}^{a_{i}^{j}}} & {\left[U_{p_{i}}\left(a_{i}^{j}, \phi_{r, p i}\right)\right]=\left(\phi_{r, p_{i}}-E\left[c_{i}^{a_{i}^{j}}\right]\right)\left(F_{v_{(2)}}\left(\phi_{r, p_{i}}\right)\right.} \\
- & \left.F_{v_{(1)}}\left(\phi_{r, p_{i}}\right)\right)+\int_{\phi_{r, p_{i}}}^{M}\left(z-E\left[c_{i}^{a_{i}^{j}}\right]\right) f_{v_{(2)}}(z) d z,
\end{aligned}
$$

Where $M$ represents the largest possible $v_{i}^{j}$. Note that the first term on the right hand side (RHS) of (11) represents the utility when the spectrum channel is leased at the reserve price. This happens if $v_{(1)}>\phi_{r, p_{i}}$ but $v_{(2)}<\phi_{r, p_{i}}$ because this channel won't be able to be leased at the second highest bid but the reserve price. Note that $\left(F_{v_{(2)}}\left(\phi_{r, p_{i}}\right)-F_{v_{(1)}}\left(\phi_{r, p_{i}}\right)\right)$ is the 
probability that this event happens. The second term on the RHS of (11) represents the expected utility when $v_{(2)} \geq \phi_{r, p_{i}}$.

Assuming that an interior maximum exists for (11), the optimal reserve price $\phi_{r, p_{i}}^{*}$ satisfies the following first-order condition of (11).

$F_{v_{(2)}}\left(\phi_{r, p_{i}}^{*}\right)-F_{v_{(1)}}\left(\phi_{r, p_{i}}^{*}\right)-\left(\phi_{r, p_{i}}^{*}-E\left[c_{i}^{a_{i}^{j}}\right]\right) f_{v_{(1)}}\left(\phi_{r, p_{i}}^{*}\right)=0$.

Thus the optimal reserve price can be determined by the above (12) if the statistical descriptions for $v_{(1)}$ and $v_{(2)}$ are available.

Similarly, in the scenarios of OSMP, if we let the lowest and second lowest acquisition costs among all effective primary users be $c_{(1)}$ and $c_{(2)}$, respectively, the expected utility gain of the secondary user $s_{i}$ with reserve price $\phi_{r, s_{i}}$ by leasing a channel from the primary users can be written as

$$
\begin{gathered}
E_{\mathbf{V}_{\mathbf{i}}, \mathbf{C}}\left[U_{s_{i}}\left(\phi_{r, s_{i}}\right)\right]=\left(E\left[v_{i}^{j}\right]-\phi_{r, s_{i}}\right)\left(F_{c_{(1)}}\left(\phi_{r, s_{i}}\right)\right. \\
\left.-F_{c_{(2)}}\left(\phi_{r, s_{i}}\right)\right)+\int_{0}^{\phi_{r, s_{i}}}\left(E\left[v_{i}^{j}\right]-z\right) f_{c_{(2)}}(z) d z .
\end{gathered}
$$

Correspondingly, the first-order condition of (13) can be obtained as follows if an interior maximum exists for (13).

$$
F_{c_{(2)}}\left(\phi_{r, s_{i}}^{*}\right)-F_{c_{(1)}}\left(\phi_{r, s_{i}}^{*}\right)+\left(E\left[v_{i}^{j}\right]-\phi_{r, s_{i}}^{*}\right) f_{c_{(1)}}\left(\phi_{r, s_{i}}^{*}\right)=0 .
$$

However, in general scenarios of spectrum allocation, each user operates only based on her/his local information and there may be no anti-cartel authorities. Thus, the number of collusive users and the number of bidding rings are unknown to each user. Consequently, even though the statistics of each user's reward payoff is available or can be estimated under homogeneous settings, the order statistics [21] of $v_{(2)}$ and $c_{(2)}$ cannot be derived without the information of the number of collusive users. Then, how to further obtain the optimal reserve prices considering the constraints in our spectrum allocation game remains unanswered.

Since our pricing game belongs to the non-cooperation games with incomplete information [18], the players need to build up certain beliefs of other players' future possible strategies to assist their decision making. In order to obtain the optimal reserve prices from (12) and (14) for robust spectrum allocation, we first derive the belief functions for primary and secondary users in the scenarios of MSOP and OSMP, respectively. Considering that there are multiple players with private information in the pricing game and what directly affect the outcome of the game are the bid/ask prices, it is more efficient to define common belief functions based on the publicly observed bid/ask prices than generating specific belief for every other player's private information. Hence, enlightened by [19], we consider the primary/secondary users' beliefs as the ratio their bid/ask being accepted at different price levels. Let $x$ and $y$ be the ask price of the primary users and the bid price of the secondary users, respectively. At each time, the ratio of asks from primary users at $x$ that have been accepted can be written as follows.

$$
\tilde{r}_{p}(x)=\frac{\mu_{A}(x)}{\mu(x)},
$$

where $\mu(x)$ and $\mu_{A}(x)$ are the number of asks at $x$ and the number of accepted asks at $x$, respectively. Similarly, at each time during the dynamic spectrum sharing, the ratio of bids from secondary users at $y$ that have been accepted is

$$
\tilde{r}_{s}(y)=\frac{\eta_{A}(y)}{\eta(y)},
$$

where $\eta(y)$ and $\eta_{A}(y)$ are the number of bids at $y$ and the number of accepted bids at $y$, respectively. Usually, $\tilde{r}_{p}(x)$ and $\tilde{r}_{s}(y)$ can be accurately estimated if a great number of buyers and sellers are participating in the pricing at the same time. However, in our pricing game, only a relatively small number of players are involved in the spectrum sharing at a specific time. The beliefs, namely, $\tilde{r}_{p}(x)$ and $\tilde{r}_{s}(y)$ cannot be practically obtained so that we need to further consider using the historical bid/ask information to build up empirical belief values.

In the scenarios of MSOP, we have the following observations: if a bid $\tilde{y}>y$ is rejected, the bid at $y$ will also be rejected; if a bid $\tilde{y}<y$ is accepted, the bid at $y$ will also be accepted. Based on the these observations, the secondary users' beliefs can be further defined as follows using the past bidding information.

Definition 1: Secondary users' beliefs: for each potential bid at $y$, define

$$
\breve{r}_{s}(y)= \begin{cases}0 & y=0 \\ \frac{\sum_{w \leq y} \eta_{A}(w)}{\sum_{w \leq y} \eta_{A}(w)+\sum_{w \geq y} \eta_{R}(w)} & y \in(0, M) \\ 1 & y \geq M\end{cases}
$$

where $\eta_{R}(w)$ is the number of bids at $w$ that has been rejected, $M$ is a large enough value so that the bids greater than $M$ will definitely be accepted. And, it is intuitive that the bid at 0 will not be accepted by any primary users.

In the scenarios of OSMP, the primary users' beliefs can be similarly derived as follows using past ask information.

Definition 2: Primary users' beliefs: for each potential ask at $x$, define

$$
\breve{r}_{p}(x)= \begin{cases}1 & x=0 \\ \frac{\sum_{w \geq x} \mu_{A}(w)}{\sum_{w \geq x} \mu_{A}(w)+\sum_{w \leq x} \mu_{R}(w)} & x \in(0, M) \\ 0 & x \geq M\end{cases}
$$

where $\mu_{R}(w)$ is the number of asks at $w$ that has been rejected. Also, it is intuitive that the ask at 0 will be definitely accepted as no cost is introduced.

Noting that it is too costly to build up beliefs on every possible bid or ask price, we can update the beliefs only at some fixed prices and use interpolation to obtain the belief function over the price space. Considering the characteristics of open ascending auction in the scenarios of MSOP, the secondary user with the highest reward payoff doesn't need to bid her/his true value to win the auction. In stead, she/he only needs to bid at the second highest possible payoff to have all other secondary users drop out of the auction. Therefore, the secondary users' belief function (17) actually represents the CDF of $v_{(2)}$. Similarly, we can obtain the CDF of $c_{(2)}$ based on the primary users' belief function (18) as $1-\breve{r}_{p}(x)$ [21].

Further, since the total number of active secondary user and the statistics of the reward payoff for each user are generally available, the PDF of $v_{(1)}$ in the scenarios of MSOP can be 
easily obtained using the order statistics in [21] as follows.

$$
F_{v_{(1)}}(x)=\prod_{i \in\{1,2, \ldots, K\}} F_{v_{i}}(x) .
$$

Also, the PDF of $c_{(1)}$ in the scenarios of OSMP can be similarly obtained as follows [21].

$$
F_{c_{(1)}}(y)=1-\prod_{i \in\{1,2, \ldots, J\}}\left(1-F_{c_{i}}(y)\right) .
$$

Therefore, the optimal reserve price for the primary user to combat user collusion in the scenarios of MSOP can be obtained from (12) using (17) and (19). Moreover, as for the scenarios of OSMP, the optimal reserve price for the secondary user can be obtained from (14) using (18) and (20).

\section{MSMP Scenarios}

In the general scenarios of MSMP, efficient collusionresistant spectrum allocation needs to be carried out among multiple primary users and secondary users while considering various user collusion patterns happening on both sides of spectrum markets, which becomes highly complicated and difficult to be analyzed. In this part, we will first derive a collusion-resistant dynamic spectrum allocation mechanism for MSMP scenarios based on the results for the OSMP/MSOP scenarios. Then, a lower bound is developed to measure the performance of the proposed mechanism by considering the extreme case of all-inclusive collusion within primary users and secondary users.

Before we derive the collusion-resistant dynamic spectrum allocation mechanism, let's discuss several upcoming challenges due to MSMP scenarios. First, the user collusion may happen not only within the primary users but also within the secondary users. The outcomes of the spectrum allocation game are determined by the collusive behaviors on both sides of the spectrum market. Second, the user collusion highly distorts the true supply and demand of spectrum resources so that the spectrum allocation efficiency will be deteriorated. It is because that except the primary user with the lowest acquisition cost and the secondary user with the highest reward payoff, the supply or demand of the spectrum resources from other users in the bidding rings will no longer be elicited through bidding process. Also, the dynamic nature of spectrum resources requires that the countermeasures to the user collusion are able to easily adapt to the spectrum dynamics by using only limited resources such as bandwidth of control channels or implementation complexity.

Consider an important property of the bidding ring in our game settings that the collusive behaviors within a bidding ring won't affect the strategies of the users who are not in the bidding ring. It means that, for instance, a primary user's optimal reserve price is only determined by the spectrum demand statistics and won't be affected by the collusive behaviors of other primary users. Similar arguments can be applied to the secondary users. Therefore, an efficient collusion-resistant dynamic spectrum allocation approach in MSMP scenarios can be similarly derived based on the results of the above discussion on the scenarios of OSMP and MSOP.
First, the definition of the beliefs of primary users and secondary users need to be redefined according to the characteristics of double auction. We have the following new observations in the scenarios of MSMP:

- If a bid $\tilde{y}>x$ is made, the ask at $x$ will also be accepted;

- If an ask $\tilde{x}<y$ is made, the bid at $y$ will also be accepted.

Based on the above observations, the users' beliefs in the scenarios of MSMP can be further refined as follows using the past bid/ask information.

Definition 3: Primary users' beliefs: for each potential ask at $x$, define

$\hat{r}_{p}(x)= \begin{cases}1 & x=0 \\ \frac{\sum_{w \geq x} \mu_{A}(w)+\sum_{w \geq x} \eta(w)}{\sum_{w \geq x} \mu_{A}(w)+\sum_{w \geq x} \eta(w)+\sum_{w \leq x} \mu_{R}(w)} & x \in(0, M) \\ 0 & x \geq M\end{cases}$

Definition 4: Secondary users' beliefs: for each potential bid at $y$, define

$\hat{r}_{s}(x)= \begin{cases}0 & y=0 \\ \frac{\sum_{w \leq y} \eta_{A}(w)+\sum_{w \leq y} \mu(w)}{\sum_{w \leq y} \eta_{A}(w)+\sum_{w \leq y} \mu(w)+\sum_{w \geq y} \eta_{R}(w)} & y \in(0, M) \\ 1 & y \geq M\end{cases}$

By using the above belief functions and the order statistics of $v_{(1)}$ and $c_{(1)}$ given the number of active primary and secondary users, the optimal reserve price for the primary user $p_{i}$ and secondary user $s_{i}$ can be obtained for MSMP scenarios as $\phi_{r, p_{i}}^{*}$ and $\phi_{r, s_{i}}^{*}$, respectively.

Before we develop the collusion-resistant dynamic spectrum algorithm, we first look at the Spread Reduction Rule (SRR) of double auction mechanisms. Generally, before the double auction pricing game converges to the equilibrium, there may exist a gap between the highest bid and lowest ask, which is called the spread of double auction. The SRR states that any ask that is permissible must be lower than current lowest ask, i.e., outstanding ask [19], and then either each new ask results in an agreed transaction or it becomes the new outstanding ask. A similar argument can be applied to bids. By defining current outstanding ask and bid as $o x$ and $o y$, respectively, we let $\bar{r}_{p}(x)=\hat{r}_{p}(x) \cdot I_{[0, o x)}(x)$ for each $x$ and $\bar{r}_{s}(y)=$ $\hat{r}_{s}(x) \cdot I_{(o y, M](y)}$ for each $y$, which are modified belief function considering the SRR. Note that $I_{(a, b)}(x)$ is defined as

$$
I_{(a, b)}(x)= \begin{cases}1 & \text { if } x \in(a, b) \\ 0 & \text { otherwise }\end{cases}
$$

By using the belief function $\bar{r}_{p}(x)$, the payoff maximization of selling the $i$ th primary user's $j$ th channel can be written as

$$
\max _{x \in(o y, o x)} E\left[U_{p_{i}}(x, j)\right]
$$

where $U_{p_{i}}(x, j)$ represents the payoff introduced by allocating the $j$ th channel when the ask is $x$, and then $E\left[U_{p_{i}}(x, j)\right]=$ $\left(x-c_{i}^{j}\right) \cdot \bar{r}_{p}(x), x>\phi_{r, p_{i}}^{*}$. Similarly, as for the secondary user $s_{i}$, the payoff maximization of leasing the $j$ th channel in the spectrum pool can be written as

$$
\max _{y \in(o y, o x)} E\left[U_{s_{i}}(y, j)\right]
$$

where $U_{s_{i}}(y, j)$ represents the payoff introduced by leasing the $j$ th channel in the spectrum pool when the bid is $y$, and then $E\left[U_{s_{i}}(y, j)\right]=\left(v_{i}^{j}-y\right) \cdot \bar{r}_{s}(y), y<\phi_{r, s_{i}}^{*}$. Therefore, by 
TABLE I

COLLUSION-RESISTANT DYNAMIC SPECTRUM ALLOCATION

\begin{tabular}{|l|}
\hline 1. Initialize the users' beliefs and bids/asks \\
$\diamond$ The primary users initialize their asks as large values close to $M$ \\
and their beliefs as small positive values less than $1 ;$ \\
$\diamond$ The secondary users initialize their bids as small values close to 0 \\
and their beliefs as small positive values less than 1 . \\
\hline 2. Belief update based on local information: \\
Update primary and secondary users' beliefs \\
using (21) and (22), respectively \\
\hline 3. Optimal reserve price for primary and secondary users: \\
Update primary users' optimal reserve prices $\phi_{r, p_{i}}^{*}$ using $(12),(19)$ and $(21) ;$ \\
Update secondary users' optimal reserve prices $\phi_{r, s_{i}}^{*}$ using (14), (20) and $(22)$. \\
\hline 4. Optimal bid/ask update: \\
$\diamond$ Obtain the optimal ask for each primary user by solving (24) given $\phi_{r, p_{i}}^{*} ;$ \\
$\diamond$ Obtain the optimal bid for each secondary user by solving (25) given $\phi_{r, s_{i}}^{*}$. \\
\hline 5. Update leasing agreement and spectrum pool: \\
$\diamond$ If the outstanding bid is greater than or equal to the outstanding ask, \\
the leasing agreement will be signed between the corresponding users; \\
$\diamond$ Update the spectrum pool by removing the assigned channel. \\
\hline 6. Iteration: \\
If the spectrum pool is not empty, go back to Step 2. \\
\hline
\end{tabular}

solving the optimization problem for each effective primary and secondary users using (24) and (25), respectively, the optimal decisions of spectrum allocation at every stage can be made conditional on dynamic spectrum demand and supply. Note that when a leasing agreement for one specific spectrum channel is achieved for a pair of primary and secondary users, the order statistics of $v_{(1)}$ and $c_{(1)}$ need to be updated as well as the optimal reserve prices for achieving the next leasing agreement. Based on the above discussions, we illustrate our collusion-resistant dynamic pricing algorithm for spectrum allocation in Table I. Note that the belief functions would help to largely decrease the pricing overhead for collusion-resistant auction-based spectrum allocation.

\section{Performance Lower Bound for MSMP Scenarios}

In order to measure the performance of the proposed collusion-resistant dynamic spectrum allocation mechanism, we derive its performance lower bound with the presence of user collusion in the following parts.

An efficient spectrum allocation scheme can be achieved by balancing the supply and demand of spectrum resources as shown in Figure 1. Thus, it is straightforward that the most inefficient spectrum allocation occurs when all the supply and demand information are concealed by the collusive behaviors of selfish users, which happens when two all-inclusive collusion are formed among the primary users and secondary users, respectively. Under this situation, the spectrum allocation game becomes a bargaining game between two players, i.e, the primary user $p_{(1)}$ with lowest acquisition cost $c_{(1)}$ and the secondary user $s_{(1)}$ with highest reward payoff $v_{(1)}$. By studying this extreme case, the lower bound of the proposed collusion-resistant scheme can be obtained.

Generally speaking, the primary user $p_{(1)}$ and secondary user $s_{(1)}$ value a spectrum channel differently so that a surplus is created. The objective of the bargaining game is to determine in which way the primary and secondary users agree to divide the surplus. Considering our bargaining game only involves two players, assume the minimal utilities that the users may obtain during the bargaining process to be
$\underline{U}_{p_{(1)}}$ and $\underline{U}_{s_{(1)}}$ for user $p_{(1)}$ and $s_{(1)}$, respectively. Let $\underline{\mathbf{U}}=\left\{\underline{U}_{p_{(1)}}, \underline{U}_{s_{(1)}}\right\}$. Assume $\overline{\mathbf{S}}$ to be a closed and convex subset of $R^{2}$, which represents the set of feasible utilities that the users can achieve if they cooperate with each other. Thus, our bargaining game between primary user $p_{(1)}$ and secondary user $s_{(1)}$ can be represented by $(\mathbf{s}, \underline{\mathbf{U}})$. Moreover, assume a bargaining solution to $(\mathbf{s}, \underline{\mathbf{U}})$ to be represented as $\varphi(\mathbf{s}, \underline{\mathbf{U}})=\left(U_{p_{(1)}}^{b}, U_{s_{(1)}}^{b}\right)$. Among all possible bargaining outcomes, the Nash Bargaining Solution [22] provides a unique and fair Pareto optimal outcome considering that the bargaining solution satisfies the following six axioms.

- Individual Rationality: $\left(U_{p_{(1)}}^{b}, U_{s_{(1)}}^{b}\right) \geq\left(\underline{U}_{p_{(1)}}, \underline{U}_{s_{(1)}}\right)$;

- Feasibility: $\left(U_{p_{(1)}}^{b}, U_{s_{(1)}}^{b}\right) \in \overline{\mathbf{S}}$;

- Pareto Optimality: If $\left(U_{p}, U_{s}\right) \in \overline{\mathbf{S}}$, and $\left(U_{p}, U_{s}\right) \geq$ $\left(U_{p_{(1)}}^{b}, U_{s_{(1)}}^{b}\right)$, then $\left(U_{p}, U_{s}\right)=\left(U_{p_{(1)}}^{b}, U_{s_{(1)}}^{b}\right)$;

- Independence of Irrelevant Alternatives: If $\left(U_{p_{(1)}}^{b}, U_{s_{(1)}}^{b}\right) \in \tilde{\mathbf{S}} \subset \overline{\mathbf{S}}$, and $\left(U_{p_{(1)}}^{b}, U_{s_{(1)}}^{b}\right)=\varphi(\overline{\mathbf{S}}, \underline{\mathbf{U}})$, then $\left(U_{p_{(1)}}^{b}, U_{s_{(1)}}^{b}\right)=\varphi(\tilde{\mathbf{S}}, \underline{\mathbf{U}})$;

- Independence of Linear Transformations: For any linear transformation $\psi, \varphi(\psi(\overline{\mathbf{S}}), \psi(\underline{\mathbf{U}}))=$ $\left(\psi\left(U_{p_{(1)}}^{b}\right), \psi\left(U_{s_{(1)}}^{b}\right)\right)$;

- Symmetry: If $\overline{\mathbf{S}}$ is invariant under all exchanges of agents and $\underline{U}_{p_{(1)}}=\underline{U}_{s_{(1)}}$, then $U_{p_{(1)}}^{b}=U_{s_{(1)}}^{b}$.

Noting that the above axioms are generally true for our bargaining game $(\mathbf{s}, \underline{\mathbf{U}})$, the corresponding Nash Bargaining Solution can be represented as follows.

$$
\begin{array}{cl}
\max _{\phi_{b}} & E_{c_{(1)}, v_{(1)}}\left[U_{p_{(1)}}\left(\phi_{b}, c_{(1)}\right) \cdot U_{s_{(1)}}\left(\phi_{b}, v_{(1)}\right)\right] \\
\text { s.t. } & G\left(U_{p_{(1)}}, U_{s_{(1)}}\right) \leq \tilde{U} \\
& U_{p_{(1)}}, U_{s_{(1)}} \geq 0
\end{array}
$$

where $U_{p_{(1)}}\left(\phi_{b}, c_{(1)}\right)=\phi_{b}-c_{(1)}$ and $U_{s_{(1)}}\left(\phi_{b}, v_{(1)}\right)=$ $v_{(1)-\phi_{b}}$. The two constraints give the feasible sets of $U_{p_{(1)}}$ and $U_{s_{(1)}}$. Note that based on the definition of linear utility functions for the users, the constraint (27) can be simplified as $U_{p_{(1)}}+U_{s_{(1)}} \leq v_{(1)}-c_{(1)}$. Therefore, the lower bound of the spectrum efficiency in the presence of user collusion can be obtained by solving (26). Moreover, after a leasing agreement is achieved between a primary user and a secondary user, the spectrum allocation continues by solving (26) with updated statistics of $v_{(1)}$ and $c_{(1)}$.

\section{Simulation Results}

In this section, we evaluate the performance of the proposed belief-assisted dynamic spectrum sharing approach in wireless networks. Considering a wireless network covering $100 \times 100$ area, we simulate $J$ primary users by randomly placing them in the network. These primary users can be the base stations serving for different wireless network operators or different access points in a mesh network. Here we assume the primary users' locations are fixed and their unused channels are available to the secondary users within the distance of 50 . Then, we randomly deploy $K$ secondary users in the network, which are assumed to be mobile devices. The mobility of the secondary users is modeled using a simplified random waypoint model [23], where we assume the "thinking time" at each waypoint is close to the effective duration of one channelleasing agreement, the waypoints are uniformly distributed 


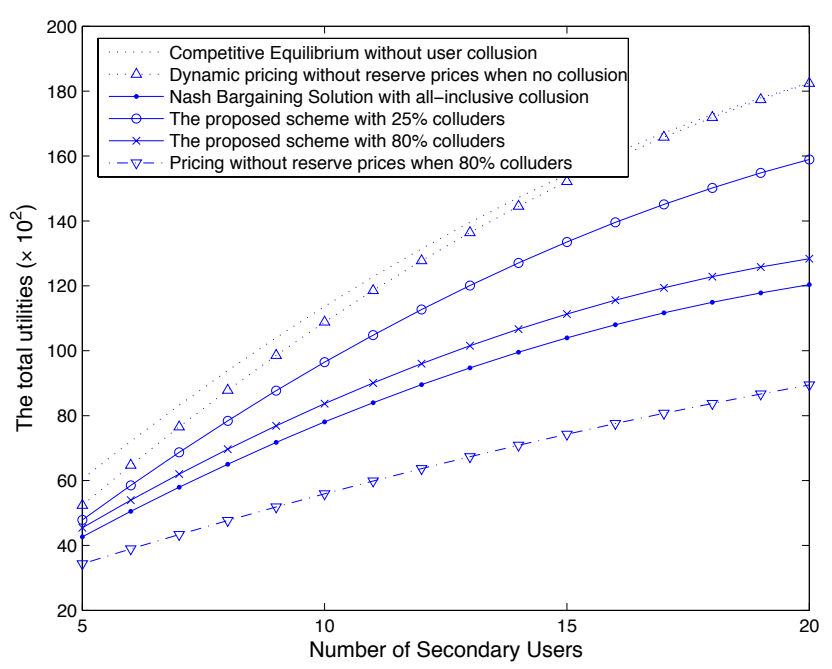

Fig. 4. Comparison of the total utilities of the CE, pricing scheme without reserve prices, and the proposed scheme with different user collusion.

within the distance of 10 , and the traveling time is much smaller than the "thinking time". Let the cost of an available channel in the spectrum pool be uniformly distributed in $[10,30]$, the reward payoff of leasing one channel be uniformly distributed in $[20,40]$. If a channel is not available to some secondary users, let the corresponding reward payoffs of this channel be 0 . Note that $J=5$ and $10^{3}$ pricing stages have been simulated. Let $n_{i}=4, \forall i \in\{1,2, \ldots, J\}$ and $\gamma=0.99$.

In Figure 4, we compare the total utilities of the competitive equilibrium, our dynamic pricing scheme with reserve prices, and our dynamic pricing scheme without reserve prices under various situations of user collusion. It can be seen from the figure that when there is no user collusion, the dynamic pricing scheme without reserve prices is able to achieve similar performance compared to the theoretical CE outcomes. Moreover, with the presence of user collusion, the proposed scheme with reserves prices achieves much higher total utilities than those of the scheme without reserve prices. Note that the total utilities increase when the number of secondary users increases. It is because that the competition among more secondary users helps to increase the spectrum efficiency. However, under the scenarios of user collusion, the performance gap between the proposed scheme with reserve price and the CE becomes greater when the number of secondary users increases. The reason is that the proposed scheme with reserve prices needs to set more strict reserve prices to combat severe user collusion when there are more secondary users. Further, the lower bound of the proposed collusion-resistant scheme shown in Figure 4 provides an efficient measurement for the maximal possible performance loss due to user collusion.

Now we study the overhead of the proposed scheme using the average number of bids and asks for each stage. In Figure 5 , the overheads of the proposed scheme with or without reserve prices are compared to those of the traditional continuous double auction when the same total utility is achieved. Assume the minimal bid/ask step $\delta$ of the continuous double auction to be 0.01 . It can be seen from the figure that our

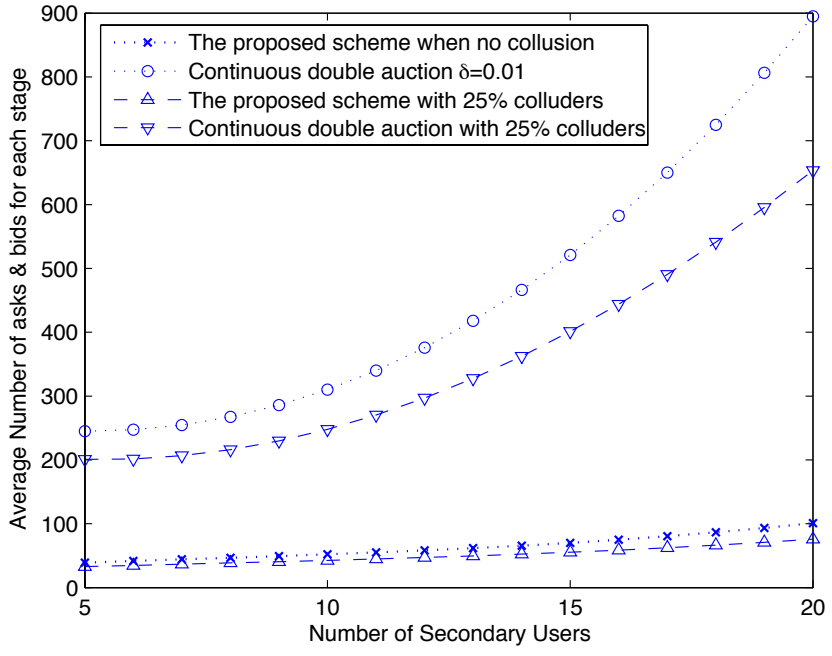

Fig. 5. Comparison of the overhead between the proposed scheme and continuous double auction scheme.

approach substantially decreases the pricing communication overhead under either the situations with user collusion or without user collusion. Note that while decreasing the overhead, our proposed approach may introduce extra complexity to update the beliefs and optimal reserve prices.

Then, we study the effect of user collusion for dynamic spectrum allocation when each secondary user is constrained by his/her monetary budget similar to [14], in which each secondary user needs to optimally allocate the budget among multiple pricing stages. For comparison, we define a static scheme in which the secondary users make their spectrumleasing decisions without considering their budget limits. Without loss of generality, we assume that the budget constraints for all secondary users are the same. By applying our proposed scheme with reserve prices to the dynamic programming approach in [14] considering budget constraints, we are able to similarly obtain the performance of the proposed collusion-resistant scheme when optimal spectrum allocation needs to be considered over time. In Figure 6, we compare the total utilities of our proposed scheme with those of the static scheme for different budget constraints when the user collusion is present. Note that the proposed collusionresistant scheme is applied to both dynamic and static pricing considering budget constraints. It can be seen from the figure that with the presence of user collusion, our proposed scheme with reserve prices achieves significant performance gains over the static scheme when the budget constraints are taken into consideration. That's because the performance loss due to the setting of reserve prices can be partly offset by exploiting the time diversity of spectrum resources among multiple sharing stages.

\section{CONCLUSIONS}

Dynamic spectrum allocation is promising for enhancing the spectrum efficiency for wireless networks. However, user collusion among selfish users severely deteriorates the efficiency of spectrum sharing. In this paper, we model the dynamic spectrum allocation as a multi-stage pricing game 


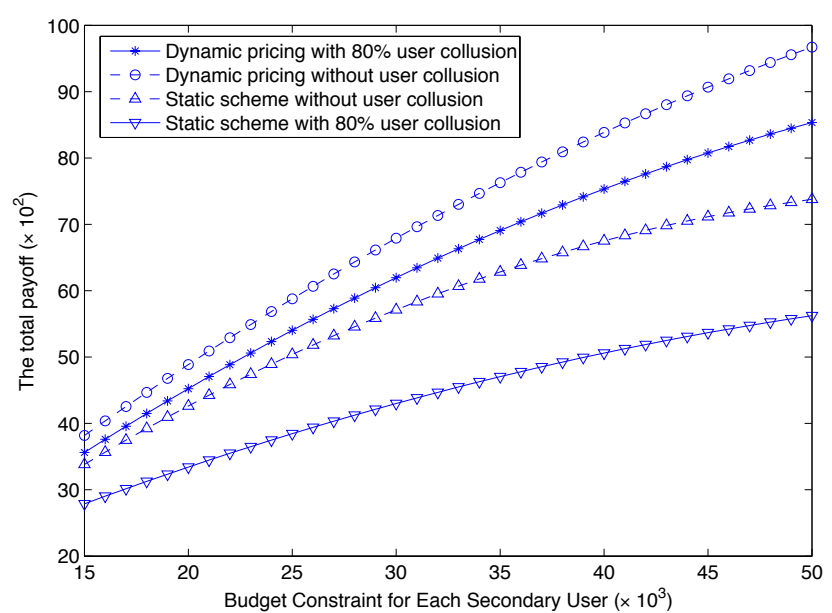

Fig. 6. Comparison of the total utilities of the proposed scheme with those of the static scheme.

and propose a collusion-resistant dynamic pricing approach to maximize the users' utilities while combating their collusive behaviors using the derived optimal reserve prices. Further, the lower bound of the proposed scheme is analyzed using Nash Bargaining Solution. Simulation results show that the proposed scheme can achieve high spectrum efficiency by only using limited overhead under various situations of user collusion.

\section{REFERENCES}

[1] DARPA XG WG, The XG Architectural Framework V1.0, 2003.

[2] I. F. Akyildiz, W. Lee, M. C. Vuran, and S. Mohanty, "Next generation/dynamic spectrum access/cognitive radio wireless networks: A survey," Computer Networks, 2006.

[3] FCC, "Facilitating opportunities for flexible, efficient, and reliable spectrum use employing cognitive radio technologies: notice of proposed rule making and order," FCC Document ET Docket No. 03-108, December 2003.

[4] R. J. Berger, "Open spectrum: a path to ubiquitous connectivity" $F C C$ ACM Queue 1, 3, May 2003.

[5] J. M. Peha, "Approaches to spectrum sharing," IEEE Commun. Mag., vol. 43, pp. 10-12, Feburary 2005.

[6] M. M. Buddhikot, "Dimsumnet: new directions in wireless networking using coordinated dynamic spectrum access," Proc. IEEE WoWMoM' 05, 2005 .

[7] C. Peng, H. Zheng, and B. Y. Zhao, "Utilization and fairness in spectrum assignment for opportunistic spectrum access," ACM Mobile Networks and Applications (MONET), May 2006.

[8] L. Cao and H. Zheng, "Distributied spectrum allocation via local bargaining," in Proc. IEEE DySPAN, 2005.

[9] R. Etkin, A. Parekh, and D. Tse, "Spectrum sharing for unlicensed bands," in Proc. IEEE DySPAN, 2005.

[10] J. Huang, R. Berry, and M. L. Honig, "Auction-based spectrum sharing," ACM Mobile Networks and Applications (MONET), pp. 405-418, 2006.

[11] M. H. Halldorson, J. H. Halpern, L. Li, V. S. Mirrokni, "On spectrum sharing games," Proc. ACM Symposium on Principle of Distributed Computing $(P O D C), 2004$.

[12] O. Ileri, D. Samardzija, and N. B. Mandayam, "Demand responsive pricing and competitive spectrum allocation via a spectrum server," Proc. IEEE DySPAN, 2005.

[13] C. Kloeck, H. Jaekel, and F. K. Jondral, "Dynamic and local combined pricing, allocation and billing system with coginitive radios," Proc. IEEE DySPAN, 2005.
[14] Z. Ji and K. J. R. Liu, "Belief-assisted pricing for dynamic spectrum allocation in wireless networks with selfish users," Proc. IEEE SECON'06, 2006.

[15] Z. Ji and K. J. R. Liu, "Collusion-resistant dynamic spectrum allocation for wireless networks via pricing," Proc. IEEE DySPAN'07, 2007.

[16] D. Fudenberg and J. Tirole, Game Theory, The MIT Press, Cambridge, Massachusetts, 1991.

[17] V. Krishna, Auction Theory, Academic Press, 2002.

[18] M. J. Osborne and A. Rubinstein, A Course in Game Theory, The MIT Press, Cambridge, Massachusetts, 1994

[19] S. Gjerstad and J. Dickhaut, "Price formation in double auctions," Games and Economic Behavior, vol. 22, pp. 1-29, 1998.

[20] Z. Ji, W. Yu, and K. J. R. Liu, "An optimal dynamic pricing framework for autonomous mobile ad hoc networks," Proc. IEEE INFOCOM, 2006.

[21] A. Papoulis, Probability, Random Variables, and Stochastic Processes, McGraw-Hill, 3rd ed., 1995.

[22] G. Owen, Game Theory, Academic Press, New York, 1991.

[23] D. B. Johnson and D. A. Maltz, "Dynamic source routing in ad hoc wireless networks, mobile computing," IEEE Trans. Mobile Computing, pp. 153-181, 2000.

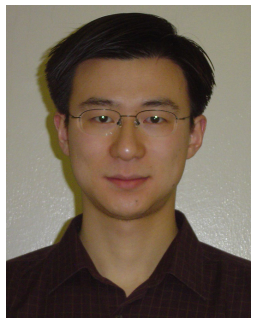

Zhu Ji received the Ph.D. degree in Electrical and Computer Engineering from University of Maryland, College Park in May 2007. He received B.S. and M.S. degrees in Electronic Engineering from Tsinghua University, Beijing, China, in 2000 and 2003 , respectively. He is currently with Qualcomm, San Diego, CA. From 2003 to 2007, he was a graduate research assistant in the Communication and Signal Processing Laboratory, University of Maryland, College Park. From 2000 to 2002, he was a visiting student (research intern) in the Wireless and Networking Group at Microsoft Research Asia, Beijing, China. His research interests are in wireless communications and networking.

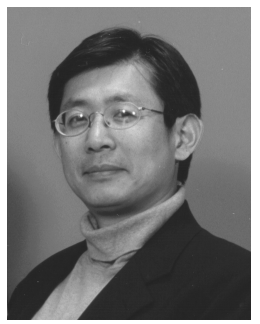

K. J. Ray Liu (F03) received the B.S. degree from the National Taiwan University and the Ph.D. degree from UCLA, both in electrical engineering. He is Professor and Associate Chair, Graduate Studies and Research, and Director of Communications and Signal Processing Laboratory of Electrical and Computer Engineering Department, University of Maryland, College Park. He leads the Maryland Signals and Information Group conducting research encompassing broad aspects of information technology including signal processing, communications, networking, information forensics and security, biomedical and bioinformatics.

Dr. Liu is the recipient of numerous honors and awards including best paper awards from IEEE Signal Processing Society (twice), IEEE Vehicular Technology Society, and EURASIP; IEEE Signal Processing Society Distinguished Lecturer, EURASIP Meritorious Service Award, and National Science Foundation Young Investigator Award. He also received various teaching and research recognitions from University of Maryland including university-level Distinguished Scholar-Teacher Award, Invention of the Year Award, Fellow of Academy for Excellence in Teaching and Learning, and college-level Poole and Kent Company Senior Faculty Teaching Award.

Dr. Liu is Vice President - Publications and on the Board of Governor of IEEE Signal Processing Society. He was the Editor-in-Chief of IEEE Signal Processing Magazine and the founding Editor-in-Chief of EURASIP Journal on Applied Signal Processing. 